\title{
Risk factors for 90-day readmission and return to the operating room following abdominal operations for Crohn's disease
} \author{
Amy L. Lightner, $\mathrm{MD}^{\mathrm{d}, *}$ \\ a Department of Colon and Rectal Surgery, Mayo Clinic, Rochester, MN \\ b Department of Visceral Surgery, Lausanne University Hospital, Lausanne, Switzerland \\ ${ }^{\mathrm{c}}$ Division of Biomedical Statistics and Informatics, Mayo Clinic, Rochester, MN \\ ${ }^{\mathrm{d}}$ Division of Colorectal Surgery, Cleveland Clinic, $\mathrm{OH}$
}

Fabian Grass, MD ${ }^{\mathrm{a}, \mathrm{b}}$, James Ansell, MD ${ }^{\mathrm{a}}$, Molly Petersen, BA', Kellie L. Mathis, MD ${ }^{\mathrm{a}}$

\section{A R T I C L E I N F O}

\section{Article history:}

Accepted 11 August 2019

Available online 20 September 2019

\begin{abstract}
A B S T R A C T
Background: This study aimed to determine timing and risk factors for 30 - and 90 -day unplanned hospital readmissions and return to the operating room.

Methods: Retrospective case series, including consecutive adult patients with Crohn's disease, undergoing a major abdominal surgical procedure during a 3.5-year inclusion period was performed. The primary outcomes were 0 - to 30-day and 30- to 90-day readmission and return to the operating room rates. Univariate and multivariable risk factors for both outcomes at 30 and 90 days were assessed through Cox regression analysis.

Results: Of 680 included patients with Crohn's disease, 89 (13.1\%) were readmitted within 30 days, 55 (8.1\%) within 30-90 days, and $11(1.6 \%)$ in both follow-up periods for a combined 90-day readmission rate of $24.4 \%(n=166)$. Multivariable risk factors for 30-day readmissions were type of procedure performed, corticosteroid use (hazard ratio [HR] $1.71, P=.01$ ), younger age (HR 0.98 per year, $P=.01$ ), and prolonged disease duration (HR 1.03 per year, $P=.03$ ). No significant risk factors identified for 30 - to 90 day readmissions. By 90 days, 76 patients (11.2\%) had a return to the operating room (of which $8.8 \%$ was within 30 days). Risk factors for 30-day return to the operating room included tobacco use (HR 1.86, $P=$ .04 ), diabetes (HR 3.30, $P=.01$ ), corticosteroid use (HR 3.51, $P<.001$ ), and preoperative immunomodulator therapy (HR 2.70, $P<.001$ ).

Conclusion: Type of surgery, corticosteroid use, younger age, and prolonged disease duration were associated with 30-day hospital readmission, and tobacco use, diabetes, corticosteroid use, and preoperative immunomodulator therapy were risk factors for 30-day return to the operating room. Postoperative biologic therapy did not increase hospital readmission or return to operating room rates within 90 days of surgery.
\end{abstract}

() 2019 Elsevier Inc. All rights reserved.

\section{Introduction}

Patients with Crohn's disease (CD) are at risk for postoperative adverse events because of immunosuppressive treatments, preponderance of malnutrition, and active disease states at the time of surgery. ${ }^{1,2}$ When looking at ileocecal resection alone, the risk of intra-abdominal sepsis after an anastomosis ranges 8\%-18\%, highlighting the severity of disease and potential for postoperative

\footnotetext{
* Reprint requests: Amy L. Lightner, MD, Associate Professor of Surgery, Department of Colorectal Surgery, Cleveland Clinic, 9500 Euclid Ave, Cleveland, OH 44195.

E-mail address: Lightna@ccf.org (A.L. Lightner).
}

morbidity. ${ }^{3-5}$ Extensive literature has sought to highlight risk factors for postoperative morbidity. Although heterogenous in study design, corticosteroids, ${ }^{6}$ biologic therapy, ${ }^{7}$ obesity, ${ }^{8}$ active tobacco use, $^{9}$ and malnutrition ${ }^{10}$ have all been associated with increased postoperative morbidity. However, despite this recognition, optimizing surgical outcomes in patients with medically refractory $C D$ remains a challenge.

Among short-term outcomes, both unplanned hospital readmissions and returns to the operating room (ROR) are critical quality metrics attributable to increased patient morbidity and significantly increased cost. ${ }^{11,12}$ Patient morbidity decreases patient satisfaction and overall quality of life. Patient readmission and ROR can more than triple the cost of an index operation. ${ }^{13,14}$ Thus, 
mitigating readmission and ROR has become a primary focus of quality-improvement projects across several hospital systems. Although there has been limited literature focused on hospital readmission and $\mathrm{ROR}$ for $\mathrm{CD}$, a recent cohort study from the Nationwide Readmission Database revealed excess charges of US\$576 million related to readmissions of patients with inflammatory bowel disease (IBD). ${ }^{15}$ Improved understanding of factors associated with readmission rates, ${ }^{15,16}$ temporal relationships from time of discharge to readmission, ${ }^{17}$ and items specific to IBD, such as the safety of biologic therapy in the immediate postoperative period, ${ }^{18}$ are important for formulating interventions to decrease postoperative inpatient hospital utilization.

This present study aimed to determine rates and reasons for unplanned hospital readmission and ROR in both the 30- and 30- to 90-day window after surgery to identify modifiable risk factors for future intervention for early and late readmissions. Furthermore, given the controversy in the safety of initiation of biologics in the perioperative period, the impact of postoperative initiation or resumption of biologic therapy on both readmission and ROR was assessed.

\section{Methods}

After we received institutional review board approval, we performed a retrospective chart review of all adult (aged $18 \mathrm{y}$ ) CD patients who underwent an elective CD-related major (general anesthesia) gastrointestinal surgical procedure at Mayo Clinic, Rochester, MN, between May 20, 2014, and December 31, 2017. The major abdominal surgical procedures included were identified by International Classification of Disease-9 (555.x) and International Classification of Disease-10 codes for CD (50.x) and Current Procedural Terminology codes for major abdominal surgery $(44120,44125$, 44130, 44140, 44141, 44143, 44144, 44145, 44146, 44147, 44150, 44151, 44155, 44156, 44157, 44158, 44160, 44180, 44187, 44188, 44202, 44204, 44205, 44206, 44207, 44208, 44210, 44211, 44212, 44227, 44310, 44314, 44316, 44320, 44340, 44345, 44346, 44615, 44620, 44625, 44626, 44640, 44650, 44660, 44661, 44799, 44950, 44790, 45110, 45111, 45112, 45113, 45114, 45119, 45120, 45136, 45395, $45397,45800,45805,45820,45825,49000$ ). Patients were excluded if they underwent an emergency operation (within $72 \mathrm{~h}$ of unplanned admission) or did not have a minimum of 90 days of traceable followup after their operation (ie, no contact established).

\section{Demographic and surgical data}

Data abstracted included demographic, disease, and surgical characteristics (patient sex, age, body mass index [BMI] category [ $<18.5$ underweight, $>30$ obese, and 18.5-30 normal weight], smoking status, duration of disease, prior intestinal resection, and predominant disease phenotype at operation). Preoperative serum laboratories within 4 weeks of surgery (leukocyte count, hemoglobin, platelet count, albumin) and within 2 weeks of surgery (Creactive protein) were retrieved. Data on preoperative medication exposure to corticosteroids and immunomodulators (azathioprine, 6-mercaptopurine, methotrexate) within 4 weeks of surgery, and biologics (infliximab, adalimumab, certolizumab pegol, vedolizumab, ustekinumab) within 12 weeks of surgery (allowing for presurgical washout given a half-life of 10-25 days for most biologics) was collected, as well as a history of biologic use and number of ongoing and past different biologics. Operative characteristics were assessed according to the operation performed and stratified as follows: ileostomy or colostomy formation, anastomosis with or without resection (ileocecectomy, segmental resection, colostomy closure, ileostomy closure, subtotal colectomy with anastomosis), resection without anastomosis (colectomy, proctectomy, proctocolectomy, ileal pouch-anal anastomosis excision with end ileostomy), local revision surgery after CD-related primary surgery (ileostomy revision or parastomal hernia repair), approach (laparoscopic versus open), anastomosis, including rate of proximal diversion. Postoperative data collection included timing (from the date of surgery) and type of new biologic therapy or resumption of previous biologic therapy, and 90-day readmission and ROR rate.

Readmission to inpatient hospital care was defined as a minimum of $24 \mathrm{~h}$ of unplanned inpatient stay after discharge from the index admission. Discharge criteria were standardized and included tolerating an oral diet, independent ambulation, adequate pain control with oral medications, and no evidence of complications requiring inpatient services by the time of dismissal. Data collected regarding readmission included timing and reasons for readmission, identified through administrative data (either through outpatient control visits or systematic phone calls at 30 and 90 days of surgery) and regrouped as follows: postoperative ileus ([POI] defined as need for nasogastric tube reinsertion), smallbowel obstruction ([SBO] defined as need for surgical reintervention), surgical infections (superficial surgical site infection [sSSI] or intra-abdominal abscess), ${ }^{19}$ surgically or radiologically confirmed anastomotic leak, medical infectious complications (including urinary tract infection and pneumonia), or bleeding complications (need for postoperative transfusion of packed red blood cells). Readmission was attributed to pain if identified as a primary complaint not attributable to any of the reasons discussed (eg, CD disease flares). Readmissions to outside facilities were tracked and stored in electronic patient charts. Reasons for readmission were double-checked through detailed chart review. ROR was defined as an unplanned reoperation under general anesthesia, directly related to the index case. The operation performed, date of operation, and indication for ROR were collected. If operations were required after the first reoperation, they were not included if related to the indication for the first reoperation.

The primary end point was the rate of readmission within 30 days and 30-90 days of the index hospital stay. Secondary end points included risk factors for 30- versus 30- to 90-day readmissions, rate of ROR at 30 days and 30-90 days of the index surgery, and reasons for readmission and ROR. When reviewing the association of readmission/reoperation after resuming or initiating (in biologic naïve patients) biologic therapy, only readmissions occurring after the exposure to a biologic were included.

\section{Statistical analysis}

Categorical variables were expressed as number (percentage) and continuous variables expressed as median (interquartile range). Comparison of demographic and clinical characteristics variables were compared among patients who restarted biologics postoperatively with those who did not using a $\chi^{2}$ test or Kruskal-Wallis test as appropriate.

Both outcomes (readmission or ROR) were assessed using Cox models. Use of biologics postoperatively was assessed as a timedependent covariate, with a patient considered as being on biologics on the date of resumption or initiation. The 90-day cumulative incidence estimates were reported as percentages with 95\% confidence interval. These estimates were displayed as cumulative incidence curves for time to readmission and ROR. Cox models were used to assess associations with readmission within 30 days (right censoring at 30 days) as well as for the period beginning on day 31 through day 90. Results of Cox models are reported as hazard ratio and 95\% confidence interval $(\mathrm{CI})$ along with the corresponding $P$ values. Variables considered in the multivariable Cox models (for both 0-30 and 30-90 days) were chosen based on the univariate significance and differed for each outcome. The multiple variable model included only patients 
Table I

Demographic and surgical details*

\begin{tabular}{|c|c|c|c|c|c|c|}
\hline & \multicolumn{6}{|c|}{ Readmission within } \\
\hline & 30 days $(N=89)$ & $30-90$ days $(N=55)$ & Both $(N=11)$ & None $(N=525)$ & Total $(N=680)$ & $P$ value \\
\hline Age at surgery, $\mathrm{y}$ & $36(24,49)$ & $34(26,49)$ & $35(28,43)$ & $40(30,54)$ & $39(29,53)$ & $.069^{*}+\dagger$ \\
\hline Sex (female) & $46(51.7)$ & $36(65.5)$ & $6(54.5)$ & $296(56.4)$ & $384(56.5)$ & $.448^{\dagger}$ \\
\hline BMI & $24(21,28)$ & $23(20,28)$ & $30(24,34)$ & $24(21,28)$ & $24(21,28)$ & $.300^{\dagger}$ \\
\hline Disease duration & $12(6,20)$ & $12(9,16)$ & $15(14,20)$ & $11(5,19)$ & $11(5,19)$ & $.650^{\dagger}$ \\
\hline Perianal disease & $31(34.8)$ & $22(40.0)$ & $2(18.2)$ & $145(27.6)$ & $200(29.4)$ & $.127^{\ddagger}$ \\
\hline Phenotype & & & & & & $.059^{\ddagger}$ \\
\hline 0 & $7(7.9 \%)$ & $7(12.7 \%)$ & $4(36.4 \%)$ & $84(16.0 \%)$ & $102(15.0 \%)$ & \\
\hline OP & $11(12.4 \%)$ & $6(10.9 \%)$ & $2(18.2 \%)$ & $50(9.5 \%)$ & $69(10.1 \%)$ & \\
\hline 3 & $27(30.3 \%)$ & $12(21.8 \%)$ & $1(9.1 \%)$ & $84(16.0 \%)$ & $124(18.2 \%)$ & \\
\hline 4 & $30(33.7 \%)$ & $18(32.7 \%)$ & $2(18.2 \%)$ & $201(38.3 \%)$ & $251(36.9 \%)$ & \\
\hline 5 & $14(15.7 \%)$ & $12(21.8 \%)$ & $2(18.2 \%)$ & $106(20.2 \%)$ & $134(19.7 \%)$ & \\
\hline Tobacco & $16(18.0)$ & $6(10.9)$ & $5(45.5)$ & $83(15.8)$ & $110(16.2)$ & .039 \\
\hline Diabetes & $3(3.4 \%)$ & $1(1.8 \%)$ & 0 & $24(4.6 \%)$ & $28(4.1 \%)$ & $.658^{\ddagger}$ \\
\hline $\mathrm{Hgb}(\mathrm{g} / \mathrm{dL})$ & $12.3(11,14)$ & $11.3(10,13)$ & $11.3(9,12)$ & $12.4(11,14)$ & $12.3(11,14)$ & $.075^{\dagger}$ \\
\hline WBC $\left(10^{6} / \mathrm{L}\right)$ & $8.2(6.1,11.0)$ & $7.6(5.7,9.4)$ & $7.9(7.4,10.9)$ & $7.5(5.8,9.4)$ & $7.6(5.9,9.6)$ & $.098^{\dagger}$ \\
\hline Platelets $\left(10^{9} / \mathrm{L}\right)$ & $307(250,423)$ & $298(240,391)$ & $351(273,404)$ & $296(231,376)$ & $299(239,382)$ & $.732^{*}, \dagger$ \\
\hline Albumin (g/L) & $3.7(3.3,4.2)$ & $3.7(3.3,4.1)$ & $3.8(3.5,3.9)$ & $4.0(3.6,4.3)$ & $3.9(3.5,4.2)$ & $.033^{\dagger}$ \\
\hline CRP $(\mathrm{mg} / \mathrm{dL})$ & $7(3,39)$ & $14(8,34)$ & $33(28,79)$ & $9(3,29)$ & $9(3,32)$ & $.201^{\dagger}$ \\
\hline Procedure group & & & & & & $.015^{\ddagger}$ \\
\hline A & $17(19.1 \%)$ & $7(12.7 \%)$ & $3(27.3 \%)$ & $48(9.1 \%)$ & 75 (11.0\%) & \\
\hline B & $43(48.3 \%)$ & $31(56.4 \%)$ & $5(45.5 \%)$ & $346(65.9 \%)$ & $425(62.5 \%)$ & \\
\hline C & $25(28.1 \%)$ & $12(21.8 \%)$ & $3(27.3 \%)$ & $95(18.1 \%)$ & 135 (19.9\%) & \\
\hline $\mathrm{D}$ & $4(4.5 \%)$ & $5(9.1 \%)$ & 0 & $36(6.9 \%)$ & $45(6.6 \%)$ & \\
\hline Laparoscopy & $40(44.9 \%)$ & $17(30.9 \%)$ & $3(27.3 \%)$ & $190(36.2 \%)$ & $250(36.8 \%)$ & $.276^{\ddagger}$ \\
\hline Anastomosis & $43(48.3 \%)$ & $31(56.4 \%)$ & $5(45.5 \%)$ & $346(65.9 \%)$ & $425(62.5 \%)$ & $.006^{\ddagger}$ \\
\hline Diversion & $1(2.3 \%)$ & $6(19.4 \%)$ & $2(40.0 \%)$ & $29(8.4 \%)$ & $38(8.9 \%)$ & $.006^{\ddagger}$ \\
\hline
\end{tabular}

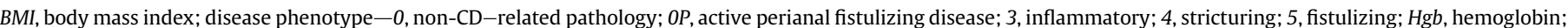


local revision surgery.

* Numbers are presented as median with interquartile rage or percentages in parentheses. Percentages should be interpreted considering time dependence.

$\dagger$ The ANOVA F-test.

$\ddagger$ The $\chi^{2}$ test.

Table II

Immunosuppression ${ }^{*}$

\begin{tabular}{|c|c|c|c|c|c|c|}
\hline & \multicolumn{6}{|c|}{ Readmission within } \\
\hline & 30 days $(N=89)$ & 30-90 days $(N=55)$ & Both $(N=11)$ & None $(N=525)$ & Total $(N=680)$ & $P$ value \\
\hline Any preoperative IMM & $32(36.0 \%)$ & $19(34.5 \%)$ & $2(18.2 \%)$ & $171(32.6 \%)$ & $224(32.9 \%)$ & $.671^{\dagger}$ \\
\hline Preoperative steroids & $32(36.0 \%)$ & $15(27.3 \%)$ & $6(54.5 \%)$ & $123(23.4 \%)$ & $176(25.9 \%)$ & $.011^{\dagger}$ \\
\hline Biologics before surgery & $81(91.0 \%)$ & $50(90.9 \%)$ & $11(100 \%)$ & $438(83.4 \%)$ & $580(85.3 \%)$ & $.070^{\ddagger}$ \\
\hline Number of prior biologics & $2(0,2)$ & $2(1,2)$ & $2(0,3)$ & $1(0,2)$ & $1(0,2)$ & $.006^{\dagger}$ \\
\hline Preoperative biologics & & & & & & $.171^{\ddagger}$ \\
\hline Adalimumab & $14(15.7 \%)$ & $6(10.9 \%)$ & $1(9.1 \%)$ & $89(17.0 \%)$ & $110(16.2 \%)$ & \\
\hline Certolizumab & $9(10.1 \%)$ & $4(7.3 \%)$ & $1(9.1 \%)$ & $31(5.9 \%)$ & $45(6.6 \%)$ & \\
\hline Infliximab & $11(12.4 \%)$ & $9(16.4 \%)$ & $3(27.3 \%)$ & $80(15.2 \%)$ & $103(15.1 \%)$ & \\
\hline Ustekinumab & $4(4.5 \%)$ & $7(12.7 \%)$ & $1(9.1 \%)$ & $23(4.4 \%)$ & $35(5.1 \%)$ & \\
\hline Vedolizumab & $22(24.7 \%)$ & $13(23.6 \%)$ & $2(18.2 \%)$ & 85 (16.2\%) & $122(17.9 \%)$ & \\
\hline None & $29(32.6 \%)$ & $16(29.1 \%)$ & $3(27.3 \%)$ & $217(41.3 \%)$ & 265 (39.0\%) & \\
\hline Type of therapy & & & & & & $.504^{\dagger}$ \\
\hline Anti-TNF & 15 (16.9\%) & $8(14.5 \%)$ & $2(18.2 \%)$ & $84(16.0 \%)$ & $109(16.0 \%)$ & \\
\hline Anti-TNF dual & $15(16.9 \%)$ & $9(16.4 \%)$ & $2(18.2 \%)$ & 103 (19.6\%) & $129(19.0 \%)$ & \\
\hline Anti-TNF triple & $4(4.5 \%)$ & $2(3.6 \%)$ & $1(9.1 \%)$ & $14(2.7 \%)$ & $21(3.1 \%)$ & \\
\hline Dual no biologic & $2(2.2 \%)$ & $1(1.8 \%)$ & 0 & $9(1.7 \%)$ & $12(1.8 \%)$ & \\
\hline Ustekinumab & $1(1.1 \%)$ & $3(5.5 \%)$ & 0 & $10(1.9 \%)$ & $14(2.1 \%)$ & \\
\hline Ustekinumab double & $2(2.2 \%)$ & $4(7.3 \%)$ & $1(9.1 \%)$ & $14(2.7 \%)$ & $21(3.1 \%)$ & \\
\hline Ustekinumab triple & $1(1.1 \%)$ & $0(0.0 \%)$ & 0 & $0(0.0 \%)$ & $1(0.1 \%)$ & \\
\hline Vedolizumab & $6(6.7 \%)$ & $5(9.1 \%)$ & $1(9.1 \%)$ & $31(5.9 \%)$ & $43(6.3 \%)$ & \\
\hline Vedolizumab dual & $14(15.7 \%)$ & $7(12.7 \%)$ & $1(9.1 \%)$ & $44(8.4 \%)$ & $66(9.7 \%)$ & \\
\hline Vedolizumab triple & $3(3.4 \%)$ & $1(1.8 \%)$ & 0 & $9(1.7 \%)$ & $13(1.9 \%)$ & \\
\hline IMM & $4(4.5 \%)$ & $1(1.8 \%)$ & 0 & $25(4.8 \%)$ & $30(4.4 \%)$ & \\
\hline Steroid & $9(10.1 \%)$ & $5(9.1 \%)$ & $2(18.2 \%)$ & $44(8.4 \%)$ & $60(8.8 \%)$ & \\
\hline None & $13(14.6 \%)$ & $9(16.4 \%)$ & $1(9.1 \%)$ & $138(26.3 \%)$ & $161(23.7 \%)$ & \\
\hline Restarted/initiated within 90 days & $36(40.4)$ & $25(45.5)$ & $4(36.4)$ & $270(51.4)$ & $335(49.3)$ & $.187^{\ddagger}$ \\
\hline Restart same biologic & $20(55.6)$ & $22(88.0)$ & $2(50.0)$ & $185(68.5)$ & $229(68.4)$ & $.050^{\ddagger}$ \\
\hline Biologic on hold because of complication & $13(14.6)$ & $6(10.9)$ & $2(18.2)$ & $8(1.5)$ & $29(4.3)$ & $<.001^{\ddagger}$ \\
\hline Time between procedure and restarting biologic (days) & $37(28-56)$ & $41(28-58)$ & $41(17-74)$ & $31(22-42)$ & $31(23-45)$ & $<.001^{\dagger}$ \\
\hline
\end{tabular}

IMM, immunomodulator; anti-TNF, antitumor necrosis factor.

* Percentages should be interpreted considering time dependence.

$\dagger$ The $\chi^{2}$ test.

$\ddagger$ The ANOVA F-test. 
Table III

Risk factors for 0- to 30-day hospital readmission

\begin{tabular}{|c|c|c|c|c|c|c|}
\hline \multirow[t]{2}{*}{ Variable } & \multirow[t]{2}{*}{ Events/total } & \multirow{2}{*}{$\frac{\text { Cumulative incidence estimates }}{\text { At } 30 \text { days }(95 \% \mathrm{Cl})}$} & \multicolumn{2}{|l|}{ Univariate Cox model } & \multicolumn{2}{|c|}{ Multivariable Cox model } \\
\hline & & & Hazard ratio $(95 \% \mathrm{CI})$ & $P$ value & Hazard ratio $(95 \% \mathrm{CI})$ & $P$ value \\
\hline Overall & $100 / 680$ & $15 \%(12-17)$ & & & & \\
\hline \multicolumn{7}{|l|}{ Sex } \\
\hline $\mathrm{F}$ & $52 / 384$ & $14 \%(10-17)$ & Reference & \multirow[t]{2}{*}{.385} & Reference & \multirow[t]{2}{*}{.540} \\
\hline M & $48 / 296$ & $16 \%(12-20)$ & $1.19(0.80-1.77)$ & & $1.13(0.76-1.70)$ & \\
\hline \multicolumn{7}{|l|}{ Tobacco } \\
\hline No & $79 / 570$ & $14 \%(11-16)$ & Reference & \multirow[t]{2}{*}{.160} & Reference & \multirow[t]{2}{*}{.235} \\
\hline Yes & $21 / 110$ & $19 \%(11-26)$ & $1.41(0.87-2.29)$ & & $1.34(0.83-2.19)$ & \\
\hline \multicolumn{7}{|l|}{ Diabetes } \\
\hline No & $97 / 652$ & $15 \%(12-17)$ & Reference & \multirow[t]{2}{*}{.538} & & \\
\hline Yes & $3 / 28$ & $11 \%(0-21)$ & $0.70(0.22-2.20)$ & & & \\
\hline \multicolumn{7}{|l|}{ Perianal Disease } \\
\hline No & $67 / 480$ & $14 \%(11-17)$ & Reference & \multirow[t]{2}{*}{.496} & Reference & \multirow[t]{2}{*}{.321} \\
\hline Yes & $33 / 200$ & $16 \%(11-21)$ & $1.16(0.76-1.76)$ & & $0.79(0.50-1.26)$ & \\
\hline \multicolumn{7}{|l|}{ Procedure group* } \\
\hline A & $20 / 75$ & $27 \%(16-36)$ & Reference & \multirow[t]{4}{*}{$<.001$} & Reference & \\
\hline B & $48 / 425$ & $11 \%(8-14)$ & $0.38(0.23-0.64)$ & & $0.40(0.23-0.70)$ & .001 \\
\hline $\mathrm{C}$ & $28 / 135$ & $20 \%(13-26)$ & $0.70(0.40-1.26)$ & & $0.80(044-1.45)$ & .468 \\
\hline $\mathrm{D}$ & $4 / 45$ & $9 \%(0-17)$ & $0.30(0.10-0.87)$ & & $0.37(0.12-1.13)$ & .081 \\
\hline \multicolumn{7}{|l|}{ Steroid } \\
\hline No & $62 / 504$ & $12 \%(9-15)$ & Reference & \multirow[t]{2}{*}{.002} & Reference & \multirow[t]{2}{*}{.012} \\
\hline Yes & $38 / 176$ & $22 \%(15-27)$ & $1.87(1.25-2.81)$ & & $1.71(1.12-2.60)$ & \\
\hline \multicolumn{7}{|l|}{ Any IMM } \\
\hline No & $66 / 456$ & $14 \%(11-17)$ & Reference & \multirow[t]{2}{*}{.704} & & \\
\hline Yes & $34 / 224$ & $15 \%(10-20)$ & $1.08(0.72-1.64)$ & & & \\
\hline \multicolumn{7}{|l|}{ Open/Lap } \\
\hline Lap & $43 / 250$ & $17 \%(12-22)$ & Reference & \multirow[t]{2}{*}{.126} & Reference & \multirow[t]{2}{*}{.282} \\
\hline Open & $57 / 430$ & $13 \%(10-16)$ & $0.73(0.49-1.09)$ & & $0.79(0.52-1.21)$ & \\
\hline \multicolumn{7}{|l|}{ Prior biologic } \\
\hline No & $8 / 100$ & $8 \%(3-13)$ & $0.49(0.24-1.00)$ & \multirow[t]{2}{*}{.051} & $0.60(0.29-1.25)$ & .172 \\
\hline Yes & $92 / 580$ & $16 \%(13-19)$ & Reference & & Reference & \\
\hline Age, y & $100 / 680$ & & $0.99(0.97-1.00)$ & .034 & $0.98(0.96-0.995)$ & .012 \\
\hline Duration of disease, $y$ & $100 / 679$ & & $1.01(0.99-1.02)$ & .504 & $1.03(1.00-1.05)$ & .029 \\
\hline BMI per 1 unit & $100 / 680$ & & $1.01(0.98-1.04)$ & .708 & & \\
\hline $\begin{array}{l}\text { Time-dependent coval } \\
\text { weeks }\end{array}$ & e time between & rocedure and restarting biologic, & $1.00(0.94-1.07)$ & .940 & $1.00(0.94-1.07)$ & .994 \\
\hline
\end{tabular}

${ }^{*}$ Procedure groups $-A$, stoma creation; $B$, anastomosis with or without resection; $C$, resection without anastomosis; $D$, local revision surgery; IMM, immunomodulatory; Lap, laparoscopy, $B M I$, body mass index.

with complete data for all predictor variables. For readmission, predefined variables were chosen based on clinical relevance and time to restarting biologics was included in the multivariable Cox model. For ROR, risk factors with $P<.11$ were included in the multivariable Cox model. The $\alpha$ level was set at $P<.05$ for statistical significance. Analyses were done using SAS v 9.4 software (SAS Institute, Cary, NC) and R v 3.4.2 software (R Core Team).

\section{Results}

In total, 32 of 712 patients (4.5\%) were lost to follow-up. Of 680 included CD patients, $166(24.4 \%)$ were readmitted within the 90 day observation period: 89 (13.1\%) within 30 days, 55 (8.1\%) within 30-90 days, and 11 (1.6\%) in both follow-up periods (within 0-30 and 30-90 days). Table I presents an overview on demographic, disease-related, and surgical items of the groups. Most patients (85.3\% of the entire cohort) had been exposed to biologic therapy preoperatively, $32.9 \%$ were exposed to immunomodulators, and $25.9 \%$ were exposed to corticosteroids. We observed no differences in medication exposure in the readmitted versus non-readmitted patients. Furthermore, exposure to immunosuppression was similar among the groups (Table II). However, differences were observed regarding preoperative corticosteroid use $(P=.01)$, number of prior biologics $(P=.006)$, and timing of postoperative biologic resumption, which occurred later in readmitted patients $(P=.0006)$.

\section{Risk factors for unplanned readmissions}

Univariate risk factors for 0- to 30-day unplanned hospital readmission were related to the type of surgery performed, with fewer readmissions occurring after local revision operations (eg, stoma revisions, parastomal hernia repairs) and procedures with an anastomosis created with or without resection, as compared with operations with new stoma creation $(P=.008)$. A patient with corticosteroids had a 30-day cumulative incidence of readmission of $22 \%$ compared with $12 \%$ in patients with no corticosteroids. Patients exposed to preoperative corticosteroids had a significantly increased risk of readmission within 30 days (hazard ratio $[\mathrm{HR}]=1.87 ; 95 \% \mathrm{CI} 1.25-2.81, P=.002$ ). On multivariable analysis, procedure type, corticosteroid use (HR 1.71; $95 \% \mathrm{CI} 1.12-2.60, P=.01$ ), young age (HR $0.98 ; 95 \% \mathrm{CI} 0.96-1$ per year, $P=.01$ ), and prolonged disease duration (HR 1.03; 95\% CI 1-1.05 per year, $P=.03$ ) were significant risk factors for 30-day readmission (Table III). No significant univariate and multivariable risk factors were identified for 30- to 90-day unplanned hospital readmissions (Table IV). Restarting biologic therapy within 90 days of surgery was not associated with an increased rate of 30-day nor 30- to 90-day readmission.

Cumulative incidence of all readmissions occurring over the 90day observation period is illustrated in Fig 1, A. Figures 1, B and 1, $C$ depict reasons for 0 - to 30-day and 30- to 90-day readmissions, respectively. The main reasons for 30-day readmissions were POI/SBO ( $n=23,23 \%$ of readmitted patients), sSSI and intra-abdominal abscess ( $n=18$ each, $18 \%$ each); whereas the primary reasons for 30 - to 90 -day 
Table IV

Risk factors for 30- to 90-day hospital readmission

\begin{tabular}{|c|c|c|c|c|c|c|}
\hline \multirow[t]{2}{*}{ Variable } & \multirow[t]{2}{*}{ Events/total } & \multirow{2}{*}{$\frac{\text { Cumulative incidence estimates }}{\text { At } 90 \text { days }(95 \% \mathrm{CI})}$} & \multicolumn{2}{|l|}{ Univariate Cox model } & \multicolumn{2}{|c|}{ Multivariable Cox model } \\
\hline & & & Hazard ratio $(95 \% \mathrm{CI})$ & $P$ value & Hazard ratio $(95 \% \mathrm{CI})$ & $P$ value \\
\hline Overall & $66 / 680$ & $10 \%(7-12)$ & & & & \\
\hline \multicolumn{7}{|l|}{ Sex } \\
\hline $\mathrm{F}$ & $42 / 384$ & $11 \%(8-14)$ & Reference & \multirow[t]{2}{*}{.250} & Reference & \multirow[t]{2}{*}{.299} \\
\hline M & $24 / 296$ & $8 \%(5-11)$ & $0.74(0.45-1.23)$ & & $0.74(0.42-1.30)$ & \\
\hline \multicolumn{7}{|l|}{ Tobacco } \\
\hline No & $55 / 570$ & $9 \%(7-12)$ & Reference & \multirow[t]{2}{*}{.889} & & \\
\hline Yes & $11 / 110$ & $10 \%(4-15)$ & $1.05(0.55-2.00)$ & & & \\
\hline \multicolumn{7}{|l|}{ Diabetes } \\
\hline No & $65 / 652$ & $10 \%(8-12)$ & Reference & \multirow[t]{2}{*}{.306} & & \\
\hline Yes & $1 / 28$ & $4 \%(0-10)$ & $0.36(0.05-2.57)$ & & & \\
\hline \multicolumn{7}{|l|}{ Perianal disease } \\
\hline No & $42 / 480$ & $9 \%(6-11)$ & Reference & \multirow[t]{2}{*}{.161} & Reference & \multirow[t]{2}{*}{.242} \\
\hline Yes & $24 / 200$ & $12 \%(7-16)$ & $1.43(0.87-2.37)$ & & $1.40(0.79-2.48)$ & \\
\hline \multicolumn{7}{|l|}{ Procedure group* } \\
\hline A & $10 / 75$ & $13 \%(5-21)$ & Reference & \multirow[t]{4}{*}{.528} & & \\
\hline B & $36 / 425$ & $8 \%(6-11)$ & $0.60(0.30-1.22)$ & & & \\
\hline $\mathrm{C}$ & $15 / 135$ & $10 \%(5-15)$ & $0.75(0.33-1.69)$ & & & \\
\hline $\mathrm{D}$ & $5 / 45$ & $11 \%(1-20)$ & $0.81(0.28-2.36)$ & & & \\
\hline \multicolumn{7}{|l|}{ Steroid } \\
\hline No & $45 / 504$ & $9 \%(6-11)$ & Reference & \multirow[t]{2}{*}{.350} & & \\
\hline Yes & $21 / 176$ & $11 \%(7-16)$ & $1.29(0.76-2.18)$ & & & \\
\hline \multicolumn{7}{|l|}{ Any IMM } \\
\hline No & $45 / 456$ & $10 \%(7-12)$ & Reference & \multirow[t]{2}{*}{.910} & & \\
\hline Yes & $21 / 224$ & $9 \%(5-13)$ & $0.97(0.58-1.63)$ & & & \\
\hline \multicolumn{7}{|l|}{ Open/Lap } \\
\hline Lap & $20 / 250$ & $8 \%(4-11)$ & Reference & \multirow[t]{2}{*}{.185} & Reference & \multirow[t]{2}{*}{.323} \\
\hline Open & $46 / 430$ & $11 \%(8-14)$ & $1.44(0.84-2.45)$ & & $1.34(0.75-2.37)$ & \\
\hline \multicolumn{7}{|l|}{ Prior biologic } \\
\hline No & $5 / 100$ & $5 \%(1-9)$ & $0.47(0.19-1.17)$ & \multirow[t]{2}{*}{.104} & $0.88(0.32-2.44)$ & \multirow[t]{2}{*}{.801} \\
\hline Yes & $61 / 580$ & $10 \%(8-13)$ & Reference & & Reference & \\
\hline Age, $y$ & $66 / 680$ & & $0.99(0.97-1.00)$ & .122 & $1.00(0.99-1.02)$ & .680 \\
\hline Duration of disease, $y$ & $66 / 679$ & & $1.00(0.98-1.02)$ & .976 & & \\
\hline BMI per 1 unit & $66 / 680$ & & $1.01(0.98-1.05)$ & .485 & & \\
\hline $\begin{array}{l}\text { Time-dependent covar } \\
\text { weeks }\end{array}$ & e time betwee & rocedure and restarting biologic, & $1.04(0.98-1.12)$ & .220 & $1.03(0.96-1.11)$ & .439 \\
\hline
\end{tabular}

${ }^{*}$ Procedure groups $-A$, stoma creation; $B$, anastomosis with or without resection; $C$, resection without anastomosis; $D$, local revision surgery; IMM, immunomodulatory; Lap, laparoscopy, BMI, body mass index.

readmissions were dehydration ( $n=20,30 \%)$, pain flares $(n=15,23 \%)$, and chronic SSSI $(n=11,17 \%)$. Out of 18 patients readmitted with pelvic abscesses, 11 needed nonoperative drain placement, and the remaining 7 patients needed surgical reintervention.

\section{Incidence and risk factors for unplanned ROR}

Overall, 76 patients (11.2\%) required an ROR within 90 days, 60 patients (8.8\%) within 30 days of primary surgery, and 16 patients (2.4\%) between 30 and 90 days. Univariate risk factors for ROR within 30 days were related to the type of surgery $(P=.05)$, perioperative corticosteroid use $(P<.0001)$, and preoperative immunomodulator therapy $(P=.008)$. Independent risk factors upon multivariable analysis for a 30-day ROR were tobacco use (HR 1.86; 95\% CI 1.03-3.36, $P=.04$ ), diabetes (HR 3.30; 95\% CI 1.27-8.56, $P=$ .01 ), corticosteroid use (HR 3.51; 95\% CI 2.08-5.92, $P<.001$ ), and preoperative immunomodulator therapy (HR 2.70; 95\% CI 1.60-4.58, $P<.001$; Online Appendix 1). We observed no significant risk factors for ROR within 30 and 90 days of surgery. Multivariable analysis was limited by the small rate of events $(n=16$; Online Appendix 2). Postoperative resumption of biologic therapy was not associated with an increased ROR within 30 days, nor within 30-90 days.

Figure 2, $A$ presents the cumulative incidence of ROR during the 90-day observation period, and reasons for reoperation are presented in Fig 2, B (0-30 days) and Fig 2, C (30-90 days). The main indications for ROR within 30 days were superficial and deep surgical site infection (SSI) $(n=23,39 \%)$, anastomotic leaks $(n=9$, $15 \%$ ), and stoma-related issues ( $n=9,15 \%$ ), with the majority of reoperations within 30-90 days were related to superficial wound issues ( $n=10,63 \%$, of which nonhealing, chronically infected wounds with failed antibiotic therapy $(n=5)$, peristomal abscesses $(n=3)$, and new onset perianal abscesses $(n=2)$. Of note, 31 of 76 patients (40.8\%) were operated during the index hospital stay and 45 of 76 patients (59.2\%) after readmission.

\section{Discussion}

Because of unfavorable patient- and disease-related conditions at the time of surgery, adverse postoperative outcomes are a major concern in $\mathrm{CD}$ patients undergoing major abdominal surgery. Furthermore, postoperative immunosuppressive therapy is being increasingly utilized in patients at high risk of disease recurrence. These features set CD patients apart from the "general" surgical population and make targeted preventive measures a prevailing matter of interest. Two relevant quality metrics from both patient and health care system economics are unplanned readmissions and reoperation. Our series of consecutive major abdominal surgeries revealed that both unplanned hospital readmissions (24.4\%) and ROR (11.2\%) were not insignificant in our series during the 90-day observation period. Furthermore, several patient-, disease-, and treatment-related risk factors were identified for 30-day readmissions and ROR, including corticosteroid use, younger age, prolonged disease duration (readmission) and tobacco use, diabetes, 


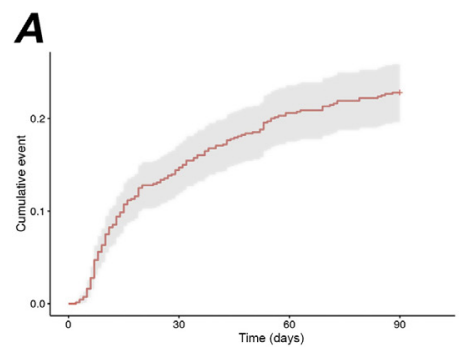

B

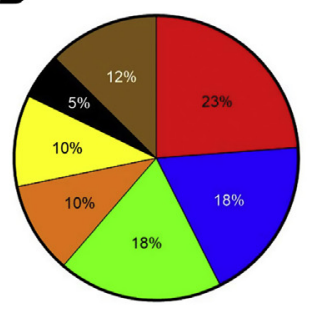

C

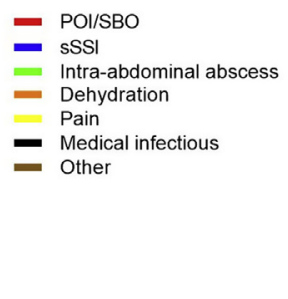

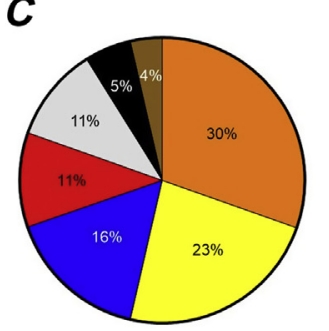

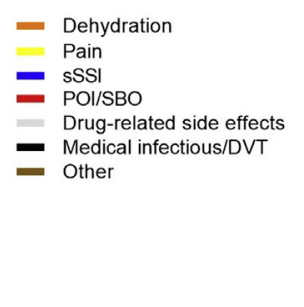

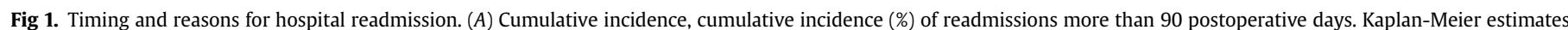

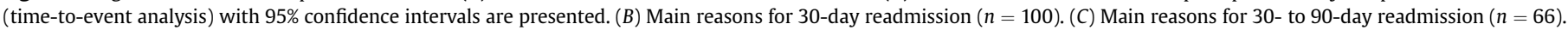
POI, postoperative ileus; SBO, small-bowel obstruction; SSSI, superficial surgical site infection; DVT, deep venous thrombosis




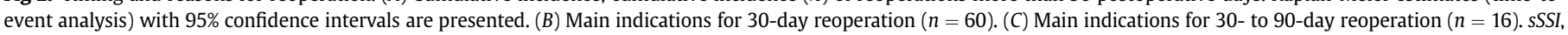
superficial surgical site infection; $S B O$, small-bowel obstruction.

corticosteroid use, and preoperative immunomodulator therapy (ROR). We identified no specific risk factors for readmission and ROR at 30-90 days postoperatively.

The 30-day readmission rate of $14.7 \%$ is within the reported range of $8 \%-19 \%$ in the literature, within similar settings. ${ }^{17,20-22}$ However, it is remarkable that $40 \%$ of unplanned readmissions in our cohort occurred between 30 and 90 days, with almost 1 out of every 4 patients seeking medical attention within 3 months of discharge. This contrasts with the findings of a recent report revealing a small number of additional admissions after 30 days of discharge. $^{21}$ This may be explained by different methodology, including systematic phone calls to track down extra-institutional readmissions. Thus, our study provides additional specifics on late readmission after the first month of discharge. Another study looking at the 90-day readmission rate, the Nationwide Readmission Database, found a similar 90-day readmission rate of $25.6 \%$ in more than 30,000 patients with $\mathrm{CD} .{ }^{15}$ Of note, in their study, a substantial proportion of patients were readmitted for psychiatryrelated conditions, including anxiety, depression, or chronic pain conditions. Therefore, the authors concluded that earlier diagnoses of chronic pain, anxiety, and depression, along with increased use of multidisciplinary teams and care transition models, may lead to decreased hospital readmissions in IBD patients.

Our analysis scrutinized reasons for readmission in 2 time frames, and reasons for readmission at 30 days and 30 to 90 days of discharge were disparate. Although most RORs (79\%) were performed within 30 days, $40 \%$ of unplanned hospital readmissions occurred between 30 and 90 days of discharge from the index hospitalization. Although early (30-day) readmissions were largely attributable to typical surgical complications, including POI and SSI, the primary reasons for late readmission between 30 and 90 days were medical issues, including dehydration and insufficient pain control. With such a substantial proportion of readmissions occurring at a 90-day window rather than a 30-day window, perhaps future studies and quality metrics should evaluate 90-day unplanned hospital readmission rates rather than 30 day readmission rates.

Several risk factors were identified for 30-day hospital readmission. First, patients with a new stoma were more likely to be readmitted, consistent with several other reports in the literature. $^{22,23}$ Ostomates are prone to dehydration, which was a predominant reason for readmission in our series (10\% at 30 days, $30 \%$ at 30-90 days). Second, young age and prolonged disease duration were both associated with increased 30-day readmission, which adds fuel to the controversial literature on the topic. Although some studies have found similar associations, ${ }^{16,24}$ a recent singleinstitution revealed more readmissions among older patients. ${ }^{21}$ Both young age and early-onset $C D$ have been associated with a more aggressive clinical course, potentially explaining the increased readmission rate in younger patients in our cohort. ${ }^{25}$ Third, our study revealed perioperative corticosteroid use not only as risk factor for readmission, but also for ROR. This is consistent with reports revealing corticosteroid use as a risk factor for a wide range of postoperative adverse events ${ }^{22,26}$ including a higher risk of postoperative wound dehiscence and infectious complications in several large-scale studies. ${ }^{27,28}$ Of note, although the type of biologic therapy did not differ among readmitted and non-readmitted patients, we did observe a trend toward more readmissions, with an increasing number of previous biologic treatments. This finding could, however, be viewed as a surrogate for disease severity, in line with former evidence. ${ }^{20}$

We found an ROR rate of $8.8 \%$ and $11.2 \%$ at 30 and 90 days, respectively. Indications for ROR were heterogenous at 30 days, including sSSI and deep SSI, anastomotic leak, stoma-related issues, and SBO, with ROR after 30 days primarily associated with sSSI. Of note, studies focusing on rates and indications for ROR in CD are scarce and have largely focused on their relationship with preoperative immunosuppressive exposure. ${ }^{29-31}$ We identified preoperative exposure to corticosteroids or immunomodulators, 
diabetes, and tobacco use were significant risk factors for an unplanned 30-day ROR. This is consistent with literature reporting increased complication rates in patients exposed to corticosteroids or preoperative immunomodulators, ${ }^{32}$ diagnosed with diabetes, ${ }^{33}$ or actively smoking. ${ }^{9}$

It was interesting to note that we did not find an association of resumption or initiation of postoperative biologic therapy and postoperative readmission or reoperation. This topic is highly debated in the current literature, because no study has specifically investigated whether resumption of biologic therapy after surgery is safe. Our results do not support the notion that postoperative resumption or initiation of biologic therapy is not safe. Biologic therapy may be safely restarted in high-risk patients, providing absence of postoperative complications preventing their resumption.

This study has several limitations. First, this is a retrospective study from a single IBD center, and the results may not be applicable to outside hospitals. The unselected cohort representing a diverse surgical activity related to $C D$ arguably leads to heterogeneity. This, however, reflects the complexity of $C D$ management and was addressed through stratification of disease presentation and surgical procedures, which was included as a confounder in the multivariable model. Furthermore, the backup role of our center may lead to a negative selection bias of patients with severe and uncontrolled disease. Second, the limited number of events impeded further subgroup analysis of outcomes of interest, such as risk factors for specific complications leading to readmission or ROR, or detailed analysis of specific preoperative drugs regarding these outcomes. Third, reasons for readmission were heterogeneous because of unselected inclusion, also of potentially unrelated causes, such as CD flares. However, both intrainstitutional and extrainstitutional readmissions were retrieved through administrative data, aiming to capture all readmissions as completely as possible, considering important, nationwide patient accrual. Finally, despite stratification for disease severity (disease phenotype, presence of perianal disease, steroid treatment, laboratory values), worse disease arguably leads to increased postoperative complication and readmission rates and potential selection bias. Causative relationships need thus to be interpreted with caution.

In conclusion, type of surgery, corticosteroid use, young age, and prolonged disease duration were identified as risk factors for 30day unplanned hospital readmissions after major abdominal surgery for Crohn's disease. Tobacco use, diabetes, and preoperative corticosteroid or immunomodulator exposure were risk factors for 30-day ROR. Weaning of preoperative corticosteroids may be a modifiable risk factor to decrease both unplanned hospital readmissions and ROR.

\section{Funding/Support}

The authors have no sources of funding or support to report.

\section{Conflict of interest/Disclosure}

Amy Lightner received consultant fees from Takeda. Edward V. Loftus, Jr, received research support from UCB, Takeda, Janssen, AbbVie, Amgen, Pfizer, Genentech, Seres Therapeutics, Receptos, Gilead, Celgene, Medimmune, and Robarts Clinical Trials. In addition, Edward V. Loftus, Jr, is a consultant for UCB; Takeda; Janssen; AbbVie; Amgen; Pfizer; Eli Lilly; Celltrion Healthcare, Ltd; and Napo Pharmaceuticals. The other authors have no related conflicts of interest to declare.

\section{Supplementary materials}

Supplementary material associated with this article can be found, in the online version, at https://doi.org/10.1016/j.surg.2019. 08.006.

\section{References}

1. Brennan GT, Ha I, Hogan C, et al. Does preoperative enteral or parenteral nutrition reduce postoperative complications in Crohn's disease patients: a meta-analysis. Eur J Gastroenterol Hepatol. 2018;30:997-1002.

2. Torres J, Mehandru S, Colombel JF, Peyrin-Biroulet L. Crohn's disease. Lancet. 2017;389:1741-1755.

3. McKenna NP, Habermann EB, Glasgow AE, Dozois EJ, Lightner AL. Intraabdominal sepsis after ileocolic resection in Crohn's disease: the role of combination immunosuppression. Dis Colon Rectum. 2018;61:1393-1402.

4. Brouquet A, Maggiori L, Zerbib P, et al. Anti-TNF therapy is associated with an increased risk of postoperative morbidity after surgery for ileocolonic Crohn disease: results of a prospective nationwide cohort. Ann Surg. 2018;267:221-228.

5. Fumery M, Seksik P, Auzolle C, et al. Postoperative complications after ileocecal resection in Crohn's disease: a prospective study from the REMIND group. Am J Gastroenterol. 2017; 112:337-345.

6. Tzivanakis A, Singh JC, Guy RJ, Travis SP, Mortensen NJ, George BD. Influence of risk factors on the safety of ileocolic anastomosis in Crohn's disease surgery. Dis Colon Rectum. 2012;55:558-562.

7. Chang MI, Cohen BL, Greenstein AJ. A review of the impact of biologics on surgical complications in Crohn's disease. Inflamm Bowel Dis. 2015;21: $1472-1477$.

8. Cai X, Shen W, Guo Z, et al. Thickness of subcutaneous fat is a predictive factor of incisional surgical site infection in Crohn's disease surgery: a retrospective study. Gastroenterol Res Pract. 2018;2018:1546075.

9. Morar PS, Hodgkinson JD, Thalayasingam S, et al. Determining predictors for intra-abdominal septic complications following ileocolonic resection for Crohn's disease-considerations in pre-operative and peri-operative optimisation techniques to improve outcome. J Crohns Colitis. 2015;9:483-491.

10. Grass F, Pache B, Martin D, Hahnloser D, Demartines N, Hubner M. Preoperative nutritional conditioning of Crohn's patients-Systematic review of current evidence and practice. Nutrients. 2017;9. https://doi.org/10.3390/nu9060562.

11. van Langenberg DR, Simon SB, Holtmann GJ, Andrews JM. The burden of inpatient costs in inflammatory bowel disease and opportunities to optimize care: a single metropolitan Australian center experience. J Crohns Colitis. 2010;4:413-421.

12. Weizman AV, Nguyen GC. Quality of care delivered to hospitalized inflammatory bowel disease patients. World J Gastroenterol. 2013;19:6360-6366.

13. Damle RN, Cherng NB, Flahive JM, et al. Clinical and financial impact of hospital readmissions after colorectal resection: predictors, outcomes, and costs. Dis Colon Rectum. 2014;57:1421-1429.

14. Wick EC, Shore AD, Hirose K, et al. Readmission rates and cost following colorectal surgery. Dis Colon Rectum. 2011;54:1475-1479.

15. Barnes EL, Kochar B, Long MD, et al. Modifiable risk factors for hospital readmission among patients with inflammatory bowel disease in a nationwide database. Inflamm Bowel Dis. 2017;23:875-881.

16. Micic D, Gaetano JN, Rubin JN, et al. Factors associated with readmission to the hospital within 30 days in patients with inflammatory bowel disease. PLoS One. 2017; 12:e0182900.

17. Mudireddy P, Scott F, Feathers A, Lichtenstein GR. Inflammatory bowel disease: predictors and causes of early and late hospital readmissions. Inflamm Bowel Dis. 2017;23:1832-1839.

18. Lightner AL. Perioperative management of biologic and immunosuppressive medications in patients with Crohn's disease. Dis Colon Rectum. 2018;61:428-431.

19. Emori TG, Culver DH, Horan TC, et al. National nosocomial infections surveillance system (NNIS): description of surveillance methods. Am J Infect Control. 1991;19:19-35.

20. White EC, Melmed GY, Vasiliauskas E, et al. Does preoperative immunosuppression influence unplanned hospital readmission after surgery in patients with Crohn's disease? Dis Colon Rectum. 2012;55:563-568.

21. Mege D, Michelassi F. Readmission after abdominal surgery for Crohn's disease: identification of high-risk patients. J Gastrointest Surg. 2018;22:1585-1592.

22. Christian KE, Jambaulikar GD, Hagan MN, et al. Predictors of early readmission in hospitalized patients with inflammatory bowel disease. Inflamm Bowel Dis. 2017;23:1891-1897.

23. Frolkis A, Kaplan GG, Patel AB, et al. Postoperative complications and emergent readmission in children and adults with inflammatory bowel disease who undergo intestinal resection: a population-based study. Inflamm Bowel Dis. 2014;20:1316-1323.

24. Nguyen GC, Bollegala N, Chong CA. Factors associated with readmissions and outcomes of patients hospitalized for inflammatory bowel disease. Clin Gastroenterol Hepatol. 2014;12:1897-18904 e1.

25. Charpentier C, Salleron J, Savoye G, et al. Natural history of elderly-onset inflammatory bowel disease: a population-based cohort study. Gut. 2014;63: $423-432$. 
26. Lopez-Sanroman A. Steroids and postoperative complications in IBD [e-pub ahead of print]. Curr Drug Targets. 2019. https://doi.org/10.2174| 1389450120666190320122939.

27. Nguyen GC, Elnahas A, Jackson TD. The impact of preoperative steroid use on short-term outcomes following surgery for inflammatory bowel disease. J Crohns Colitis. 2014;8:1661-1667.

28. Lichtenstein GR, Feagan BG, Cohen RD, et al. Serious infection and mortality in patients with Crohn's disease: More than 5 years of follow-up in the TREAT registry. Am J Gastroenterol. 2012;107:1409-1422.

29. Law CCY, Narula A, Lightner AL, McKenna NP, Colombel JF, Narula N. Systematic review and meta-analysis: preoperative vedolizumab treatment and postoperative complications in patients with inflammatory bowel disease. J Crohns Colitis. 2018;12:538-545.
30. Lightner AL, McKenna NP, Tse CS, et al. Postoperative outcomes in ustekinumab-treated patients undergoing abdominal operations for Crohn's disease. J Crohns Colitis. 2018;12:402-407.

31. Rosenfeld G, Qian H, Bressler B. The risks of post-operative complications following pre-operative infliximab therapy for Crohn's disease in patients undergoing abdominal surgery: A systematic review and meta-analysis. J Crohns Colitis. 2013;7:868-877.

32. Yu CS, Jung SW, Lee JL, et al. The influence of preoperative medications on postoperative complications in patients after intestinal surgery for Crohn's disease. Inflamm Bowel Dis. 2019;25:1559-1568.

33. Seminerio JL, Koutroubakis IE, Ramos-Rivers C, et al. Impact of obesity on the management and clinical course of patients with inflammatory bowel disease. Inflamm Bowel Dis. 2015;21:2857-2863. 